\title{
User Knowledge, Data Modelling, and Visualization: Handling through the Fuzzy Logic-Based Approach
}

\author{
Xiaoqun Liao, ${ }^{1}$ Shah Nazir $\mathbb{D},{ }^{2}$ Yangbin Zhou $\mathbb{D},{ }^{3}$ Muhammad Shafiq, ${ }^{4}$ and Xuelin $\mathrm{Qi}^{3}$ \\ ${ }^{1}$ Information and Network Center, Xi'an University of Science and DSTechnology, Xi'an 710054, China \\ ${ }^{2}$ Department of Computer Science, University of Swabi, Swabi, Pakistan \\ ${ }^{3}$ State Grid Tibet Electric Power Co., Ltd, Lhasa, 850000, China \\ ${ }^{4}$ Cyberspace Institute of Advance Technology, Guangzhou University, Guangzhou, China \\ Correspondence should be addressed to Yangbin Zhou; mary@xust.edu.cn
}

Received 9 October 2020; Revised 11 November 2020; Accepted 16 November 2020; Published 26 February 2021

Academic Editor: M. Irfan Uddin

Copyright $\odot 2021$ Xiaoqun Liao et al. This is an open access article distributed under the Creative Commons Attribution License, which permits unrestricted use, distribution, and reproduction in any medium, provided the original work is properly cited.

\begin{abstract}
In modern day technology, the level of knowledge is increasing day by day. This increase is in terms of volume, velocity, and variety. Understanding of such knowledge is a dire need of an individual to extract meaningful insight from it. With the advancement in computer and image-based technologies, visualization becomes one of the most significant platforms to extract, interpret, and communicate information. In data modelling, visualization is the process of extracting knowledge to reveal the detail data structure and process of data. The proposed study aim is to know about the user knowledge, data modelling, and visualization by handling through the fuzzy logic-based approach. The experimental setup is validated through the data user modelling dataset available in the UCI web repository. The results show that the model is effective and efficient in situations where uncertainty and complexity arise.
\end{abstract}

\section{Introduction}

With the development of modern day technologies, user knowledge is increasing day by day. Users are trying to capture the essential information from the domain knowledge which is large in volume. Capturing the important information is toward the success for smooth functionality of user knowledge. Data exist in different forms such as structured and unstructured. The big data are the data whose scale, diversity, and complexity need new algorithms, structures, techniques, and analytics for the managements and visualizations and to pull out hidden information [1]. According to IDC [2], in late 2011, about $1.8 \mathrm{ZB}$ of data was created as of that year. Worldwide, electronic data of approximately $1.2 \mathrm{ZB}\left(10^{21}\right)$ are generated per year by different sources [3]. By 2020, 40 ZB data is expected [4].

Visual contexts are made from the visualization of user knowledge and data to translate the information through graphs or maps for making data easier for humans. In visualization, patterns are identified from a large bulk of data and are plotted through information visualization, graphics, and statistical graphics. Data visualization is one of the processes of data science in which data are collected, modelled, and processed, so the visualization should be in order to draw conclusions from the data. Visualization of data has importance in every field of life. It can be used in teaching, healthcare, artificial intelligence, big data, and many others to share the extracted information with the stockholders.

Knowledge, data, and information are widely used in visualization in interrelated perspectives. Visualization indicates diverse stages of understanding and abstraction. Visualization aim is to gain meaningful insights from the data [5]. Advancements in visualization bring more venture reporting.

Through data visualization, one can interact with data and go for analysis. Several benefits can be gained from 
visualization of data, such as efficient way to communicate, concrete, and abstract the message and innovative approaches for scientific and engineering purposes. Visualization of information is "the graphical presentation of abstract data" which "attempts to reduce the time and the mental effort users need to analyze large datasets." [6]

The contribution of the proposed research is to know about the user knowledge, data modelling, and visualization by handling with applications of the fuzzy logic-based approach. The experimental setup of the proposed research is validated through the data user modelling dataset available in the UCI web repository [7]. The following are the key concepts of the proposed research:

(i) To know about the user knowledge, data modelling, and visualization

(ii) To use the fuzzy logic-based approach for handling user knowledge, data modelling, and visualization

(iii) To visualize the dataset in order to get meaningful insights

(iv) To validate the work by using the "data user modelling dataset"

The organization of the paper is as follows: Section 2 represents the related work regarding user knowledge, data modelling, and visualization with different literature studies. Section 3 shows the research method and modelling of the proposed study with the detail of visualization of the dataset. Section 4 discusses the results and discussion section of the paper. The paper is concluded in Section 5.

\section{Related Work}

Different approaches, tools, and techniques are used in practice for user knowledge, data modelling, and visualization. Sahu and Dwivedi [8] proposed an approach of knowledge transfer by the domain-independent use latent factor for cross-domain recommender systems. The method used tr-factorization. The authors in [9] studied the usage of media effects in online commentaries on creating knowledge. The user groups were divided into three categories: passive participants, active participants, and bystander. Their experimental results revealed that the active participants largely tend to use tablet PC and smartphones for the creation of knowledge in the online space. Flowers and Meyer [10] focused on the user knowledge value for entrepreneurs and tackled the gap in the literature associated to the activities of entrepreneurs and user knowledge in the digital services. The framework of Innovation Opportunity Space was proposed and applied on a UK-based mobile telephony supplier Giffgaff for the issues faced by the user knowledge application to digital services. Constant [11] extracted important insights from the crystal's geometry and physical properties for the creation of new structuring according to the methodology of knowledge-visualization. Desimoni and Po [12] presented the analysis of the state-of-the-art tools for the visualization of linked data. Full list of 77 linked data visualization tool in the previous research and integrating new tools published recently online. Based on usability and their features, the visualization tools are compared and described.

Huang et al. [13] explored the cognitive approach for following the user-centred process in visualization graphs. A graph-based visualization model was proposed which is a two-stage conceptualized assessment cycle. Silva et al. [14] proposed a solution of visual analytics based on the use of several coordinate views for the description of diverse aspects of ontology and the technique of degree of interest use for reduction of complexity in the visual representation of ontology. Yu and Shi [15] presented a mini survey consisting of the user-based taxonomy that converts the works of the state of the art in the field. Luo [16] investigated how cognitive style, task difficulty, and spatial ability affect choice and preference of the visualization format and how the visualization selected affects the confidence and decision accuracy. Gebremeskel and Biazen [17] designed a system which is able to analyze and handle large-scale data. The authors in [18] presented TrajAnalytics, open-source software, for modelling, transforming, and visualizing the urban trajectory data for the study of urban and transportation. The approach allows practitioners to understand the data of the population mobility and find out knowledge. A conceptual model for data is presented which incorporates the geostructure with trajectory data with the help of different access queries of data.

Rojas and Villegas presented an approach of representation and scheme of investigative visualization for the decision tree in the knowledge discovery database process for data mining [19]. Macek and Atzmueller [20] presented a new concept of visualization for the user history interactions. Association rules are derived and visualized through heatmaps. The impact of the approach is demonstrated by real-world examples of data such as Twitter dump of 2009. Giunchiglia et al. [21] proposed the SemUI tool-based solution as the multitiered method consists of the (a) semantic layer which incorporates data through the notion of the entity of the real world and groups them based on their differences and similarities and (b) layer of visualization which concurrently shows several views based on entity properties. The authors in [22] presented an approach of visual analytics for the visual data mining and interactive machine learning. In the approach, techniques of multidimensional data visualization are applied for the facilitation of user interactions with machine learning and data mining process. Rafi [23] proposed a multidimensional interface for adopting the resource space model and presented its advantages in the property letting application. The authors in [24] presented 
a methodology for exploiting visual language CoDe based on the logic paradigm. CoDe gives the structure for organizing visualization by the CoDe model and represents the relationships between items of the information graphically. The authors in [25] proposed a model of visual analytic knowledge generation to tie different frameworks.

\section{Research Methodology}

The following sections briefly explain the methodology section of the proposed research.

\subsection{Library-Based Search to Show the Status of the Existing} Research. Different libraries were searched for identifying relevant information regarding the user knowledge, data modelling, and visualization. The purpose was to identify information such as the number of publications in the given year, publication topics, publication disciplines, publication title, and type of publications. For the search process, famous libraries such as IEEE, ScienceDirect, Springer, Tailor \& Francis, Wiley online library, and MDPI were searched for showing the relevant information. This information was presented in the form of different figures. Figure 1 represents the type of publications in the IEEE library.

Figure 2 represents the conference locations where the conferences were held.

Figure 3 represents the topics of publications along with the total number of publications.

Figure 4 represents the publication years and the number in the ScienceDirect library.

Figure 5 represents the article types with the publication number.

Figure 6 represents the publication title with the total number of publications.

Figure 7 represents the article types along with the paper number in the Springer library.

Figure 8 represents the content types along with the total number of publications.

Figure 9 represents the publication topic with the number of papers.

Figure 10 represents the number of publications published in the given languages.

Figure 11 represents the discipline along with the number of publications in the Tailor \& Francis library.

Figure 12 represents the discipline along with the number of publications in the Wiley online library.

Figure 13 represents the discipline of publication in the MDPI library.

3.2. Visualization of the Dataset. The purpose of user knowledge and visualization is to extract meaningful insights from the knowledge and present it in an organized form to be easily understood and analysed. The experimental setup of the proposed research is validated through the data user modelling dataset available in the UCI web repository [7]. Visualization of the class distribution of the dataset was done to plot each class which is very low, low, middle, and high. The purpose of this visualization is to show the dataset which is easily understandable to the reader as compared to study the actual dataset. Figure 14 represents the visualization of class distribution "high".

Figure 15 represents the visualization of class distribution "very_low."

Figure 16 represents the visualization of class distribution "middle."

Figure 17 represents the visualization of class distribution "low."

3.3. User Knowledge, Data Modelling, and Visualization through the Fuzzy Logic. Visualization is to give a meaningful structure to huge data and to extract the significant information from them. With the help of visualization, information can be mined for the extraction of meaningful insights for the analysis under consideration. The important terminologies used for visualization are shown in Figure 18.

The fuzzy logic (FL) is a tool used to solve a situation of vagueness and uncertainty. It was developed by A. Z. Lofti in 1965 [26, 27]. FL has several applications in different fields of real life such as in control system, washing machines, transmission system of cars, vacuum cleaner, and software system [28-32]. The fuzzy inference system used in the proposed research makes it simple to structure the facts and provide a way out for the vague information. For more details regarding the fuzzy concept, refer Zadeh [26].

The concept of fuzzy logic was used for user knowledge, data modelling, and visualization of the proposed study. Initially, different inputs STG, SCG, STR, LPR, PEG were plotted with the membership functions (mf) low, medium, and high. The output UNS was plotted with the mf very low, low, medium, high, and very high. Figure 19 shows the input, output, and $\mathrm{mf}$ plotting process of the proposed model.

After plotting the inputs, output, and $\mathrm{mf}$, rules were made to model the proposed system of user knowledge, data modelling, and visualization. Figure 20 shows the rule editor of the proposed system.

Different rules were designed from the $\mathrm{mf}$. Some of these rules are given in the following:

(1) If (STG is Medium) and (SCG is Low) and (STR is Low) and (LPR is Low) and (PEG is Low) then (UNS is Very_low) (0.1)

(2) If (STG is Medium) and (SCG is Medium) and (STR is Low) and (LPR is Low) and (PEG is Low) then (UNS is Very_low) (0.2) 


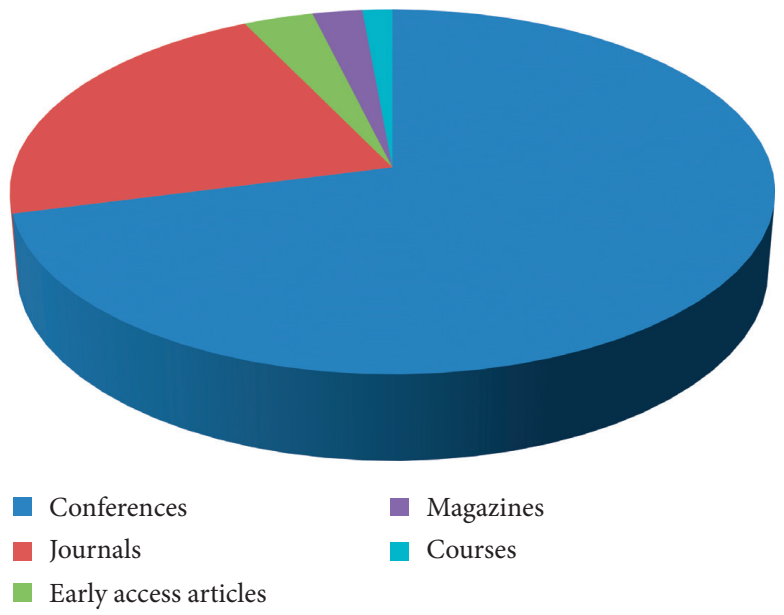

Figure 1: Publication type.

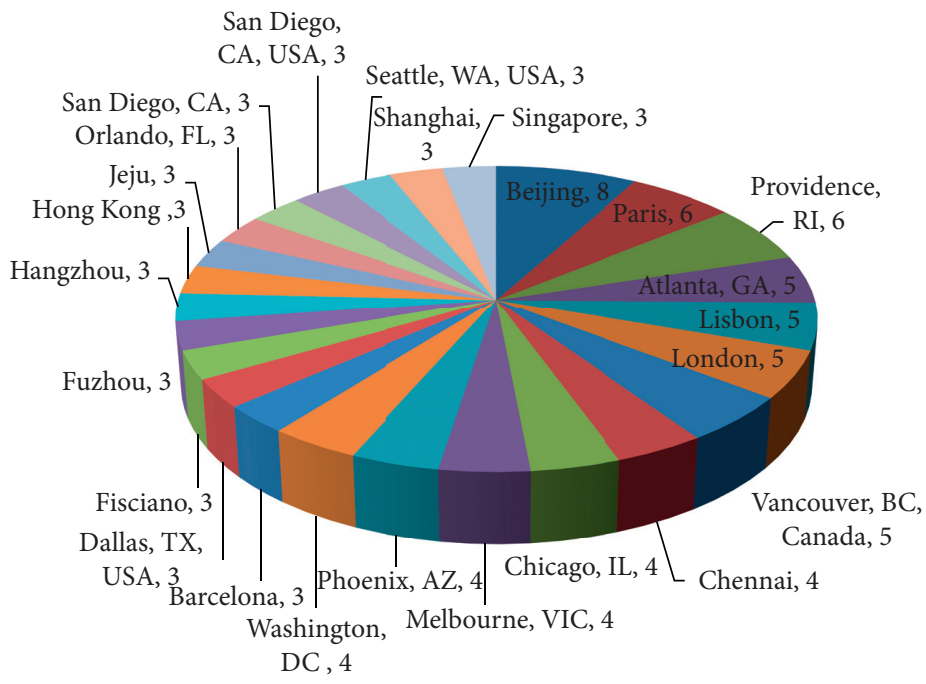

FIgURe 2: Conference locations.

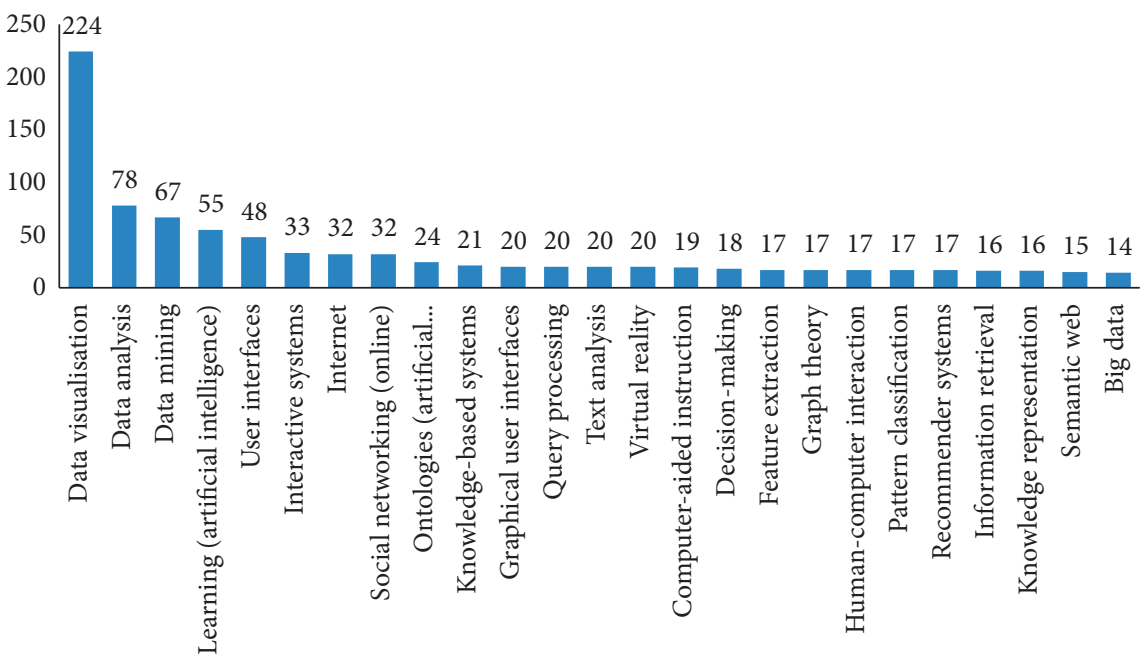

FIgURE 3: Topics with the number of publications. 


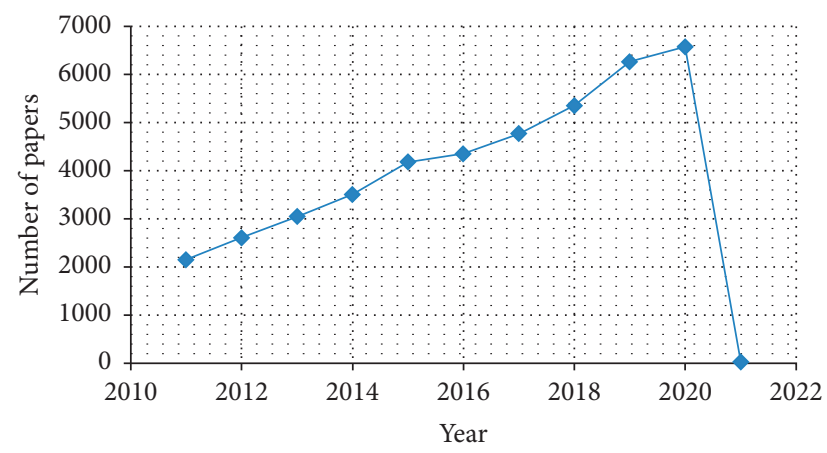

Figure 4: Years and the number of publications.

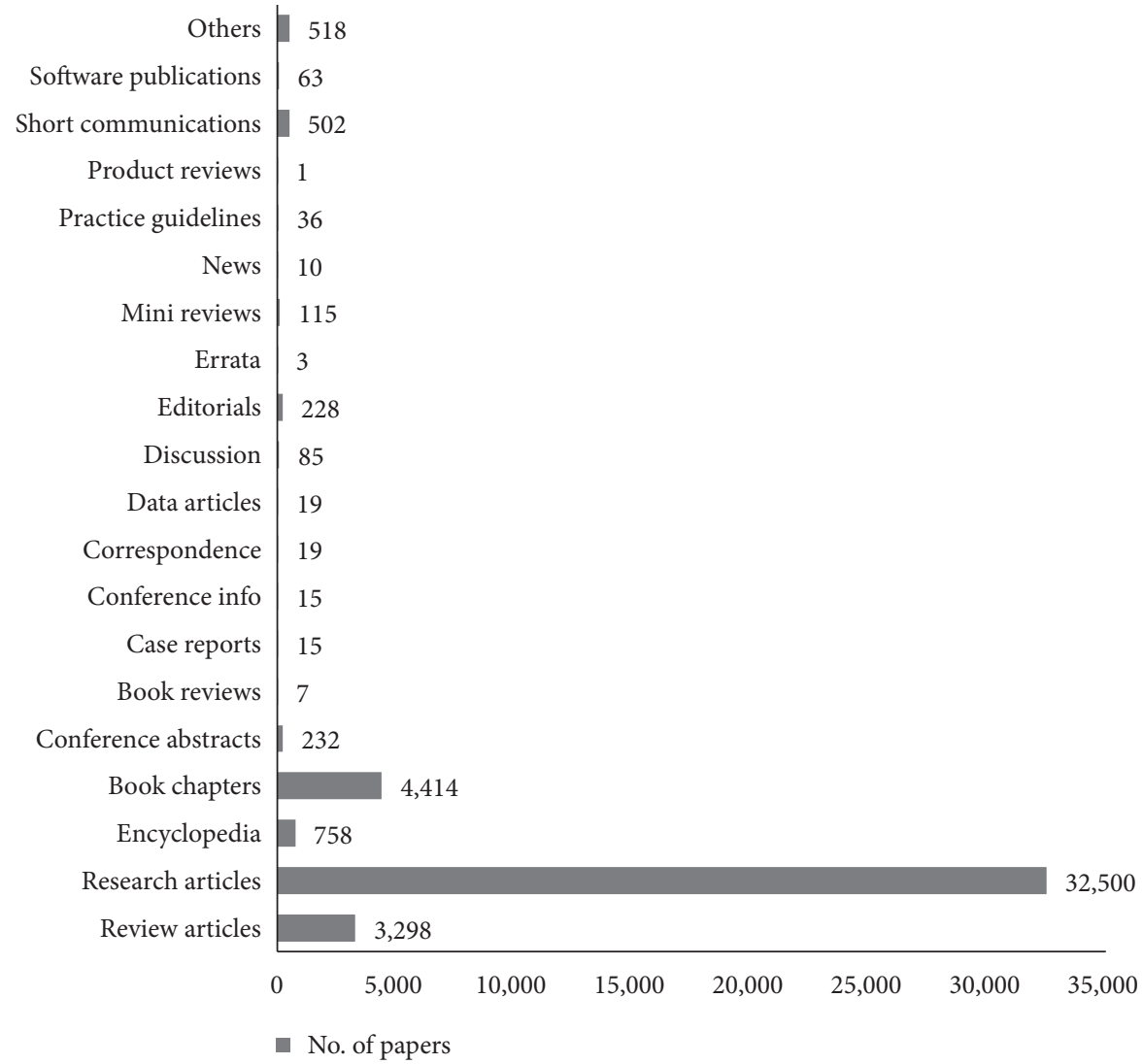

FIGURE 5: Article types with the total number of publications.

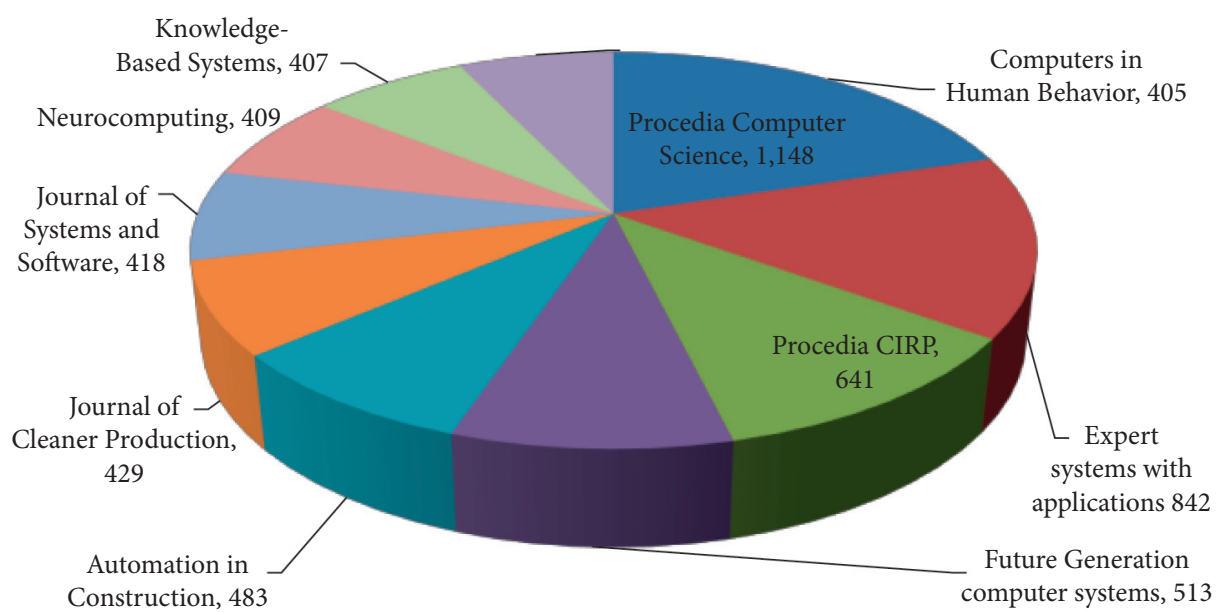

Figure 6: Publication title with the total number of publications. 


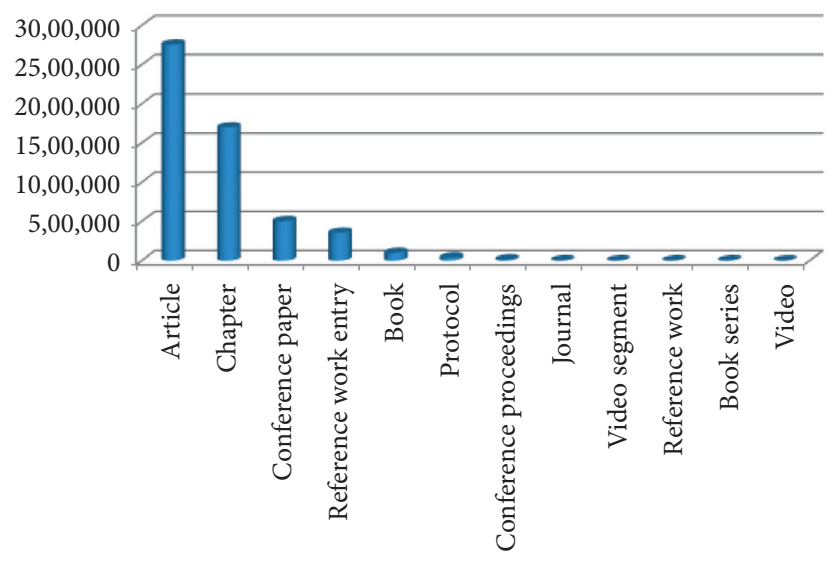

Figure 7: Article types along with the total number of papers.

\begin{tabular}{|lr|}
\hline Content type & \\
\hline Article & $2,752,396$ \\
\hline Chapter & $1,695,245$ \\
\hline Conference paper & 499,271 \\
\hline Reference work entry & 351,908 \\
\hline Book & 96,120 \\
\hline Protocol & 36,687 \\
\hline Conference proceedings & 12,004 \\
\hline Journal & 2,476 \\
\hline Video segment & 1,154 \\
\hline Reference work & 1,063 \\
\hline Book series & 1,047 \\
\hline Video & 122 \\
\hline
\end{tabular}

FIGURE 8: Type of content with the publication number.

(3) If (STG is Medium) and (SCG is Medium) and (STR is Medium) and (LPR is High) and (PEG is High) then (UNS is Very_high) (0.8)

(4) If (STG is High) and (SCG is Low) and (STR is Low) and (LPR is High) and (PEG is High) then (UNS is High) (0.61)

(5) If (STG is Low) and (SCG is Low) and (STR is High) and (LPR is High) and (PEG is High) then (UNS is High) (0.7)

(6) If (STG is Medium) and (SCG is Medium) and (STR is Medium) and (LPR is High) and (PEG is High) then (UNS is High) (0.8)
The obtained model from the designing of rules is shown in Figure 21.

\section{Results and Discussion}

The fuzzy inference system was designed for handling user knowledge, data modelling, and visualization. The purpose of this study was to plot and visualize the user knowledge and data modelling and to present the concept of visualization to present the data in a meaningful form for the reader. The model was designed from different inputs, $\mathrm{mf}$, and output. Model description of the designed model is given in Table 1. 


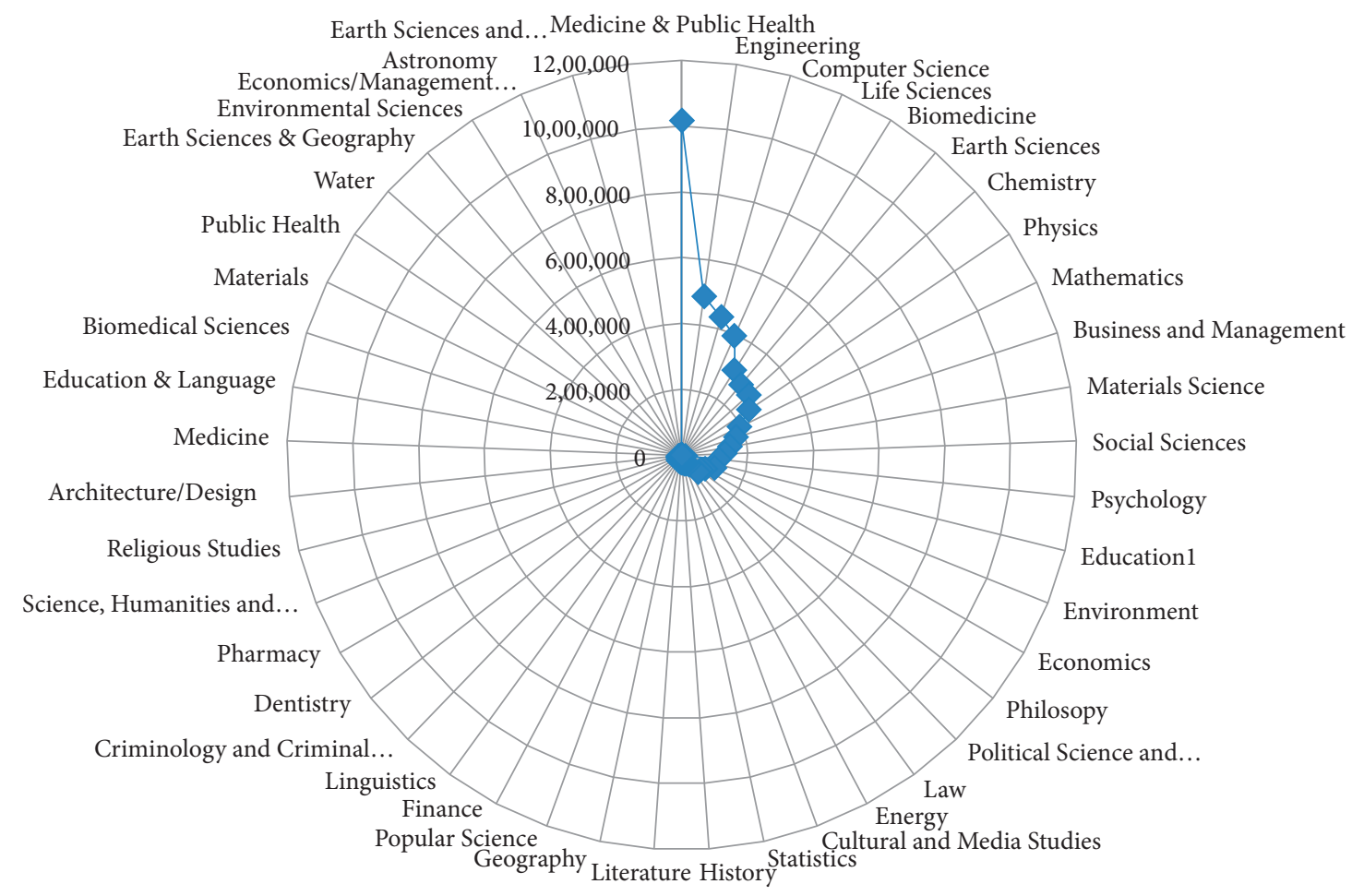

FIGURE 9: Publication topic with the number of papers.

45,00,000

Figure 10: Number of publications published in the given languages.

The fuzzy system was obtained in order to pass the inputs. The following is the structure of the proposed system:

$$
>\text { fismat }=\text { readfis }
$$

fismat $=$

$$
\text { name: "fuzzy system" }
$$

type: "mamdani"

andMethod: "min"

orMethod: "max"

defuzzMethod: "centroid"
impMethod: "min"

aggMethod: "max"

input: $[1 \times 5$ struct $]$

output: $[1 \times 1$ struct $]$

$>>a=$ readfis

$a=$

name: "fuzzy system"

type: "mamdani"

andMethod: "min"

orMethod: "max" 


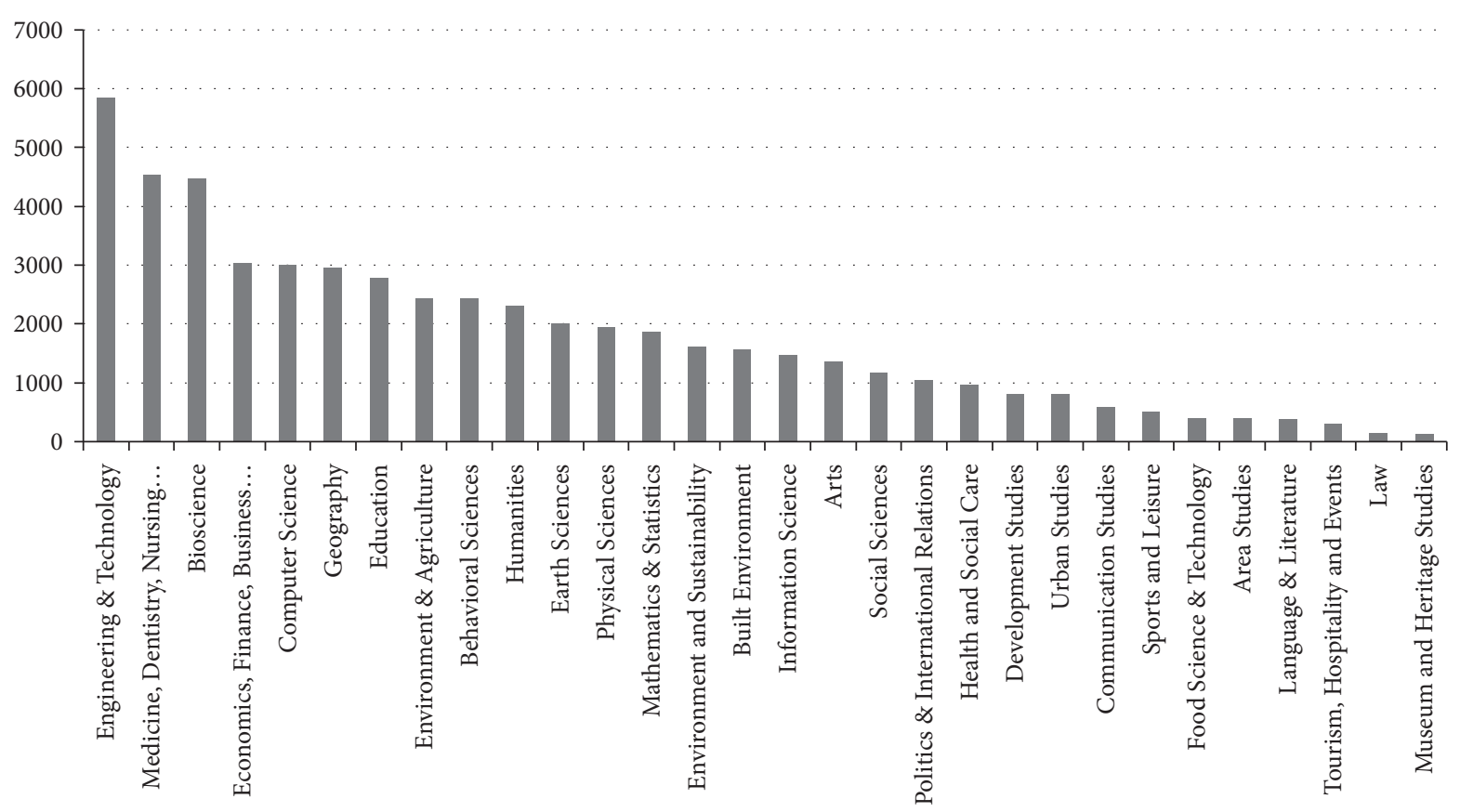

FIGURE 11: Discipline along with the number of publications.

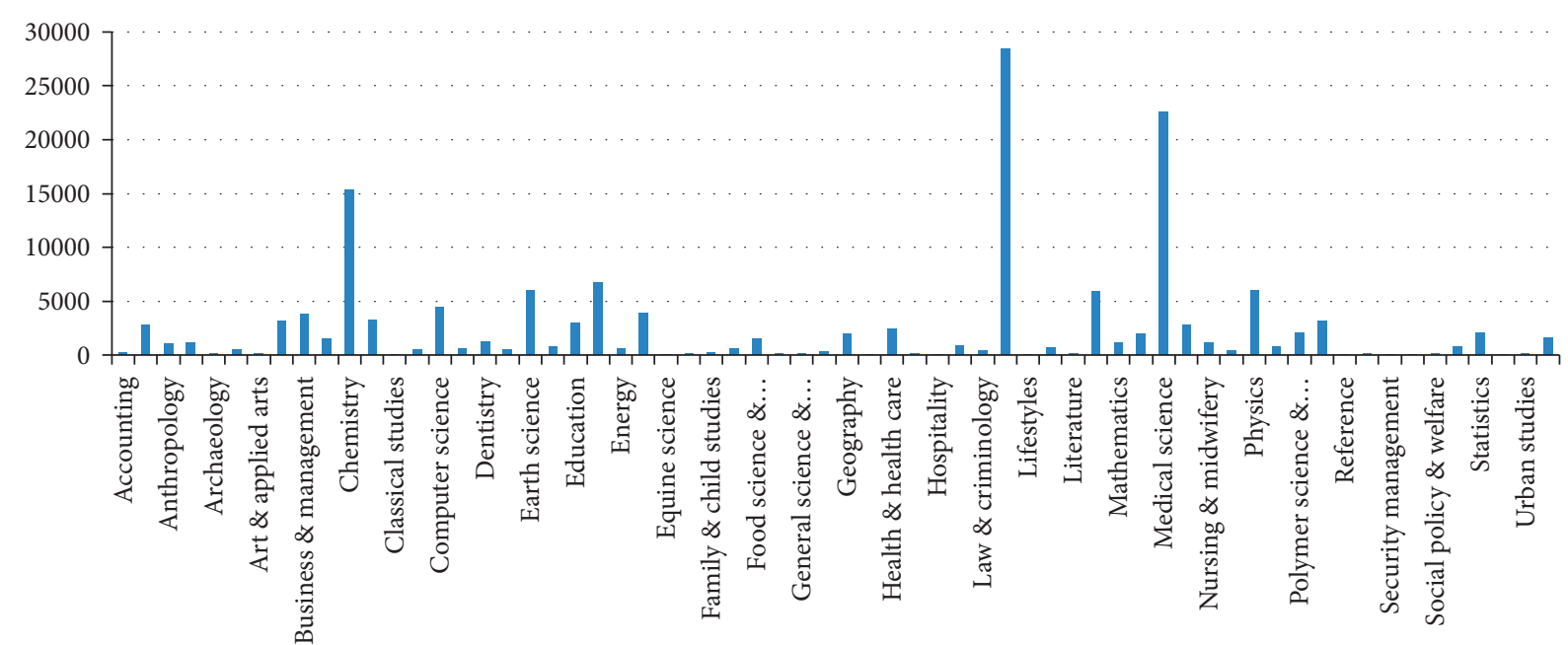

FIgURE 12: Discipline along with the number of publications in the Wiley online library.

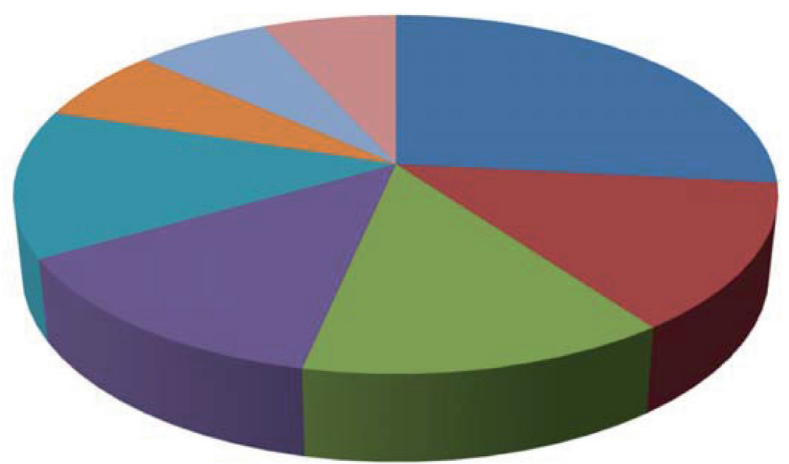

- Environmental \& Earth Sciences

- Physical Sciences

- Chemistry \& Materials Science

- Biology \& Life Sciences

- Computer Science \& Mathematics

- Business \& Economics

- Engineering

- Medicine \& Pharmacology

FIGURE 13: Discipline of publication. 


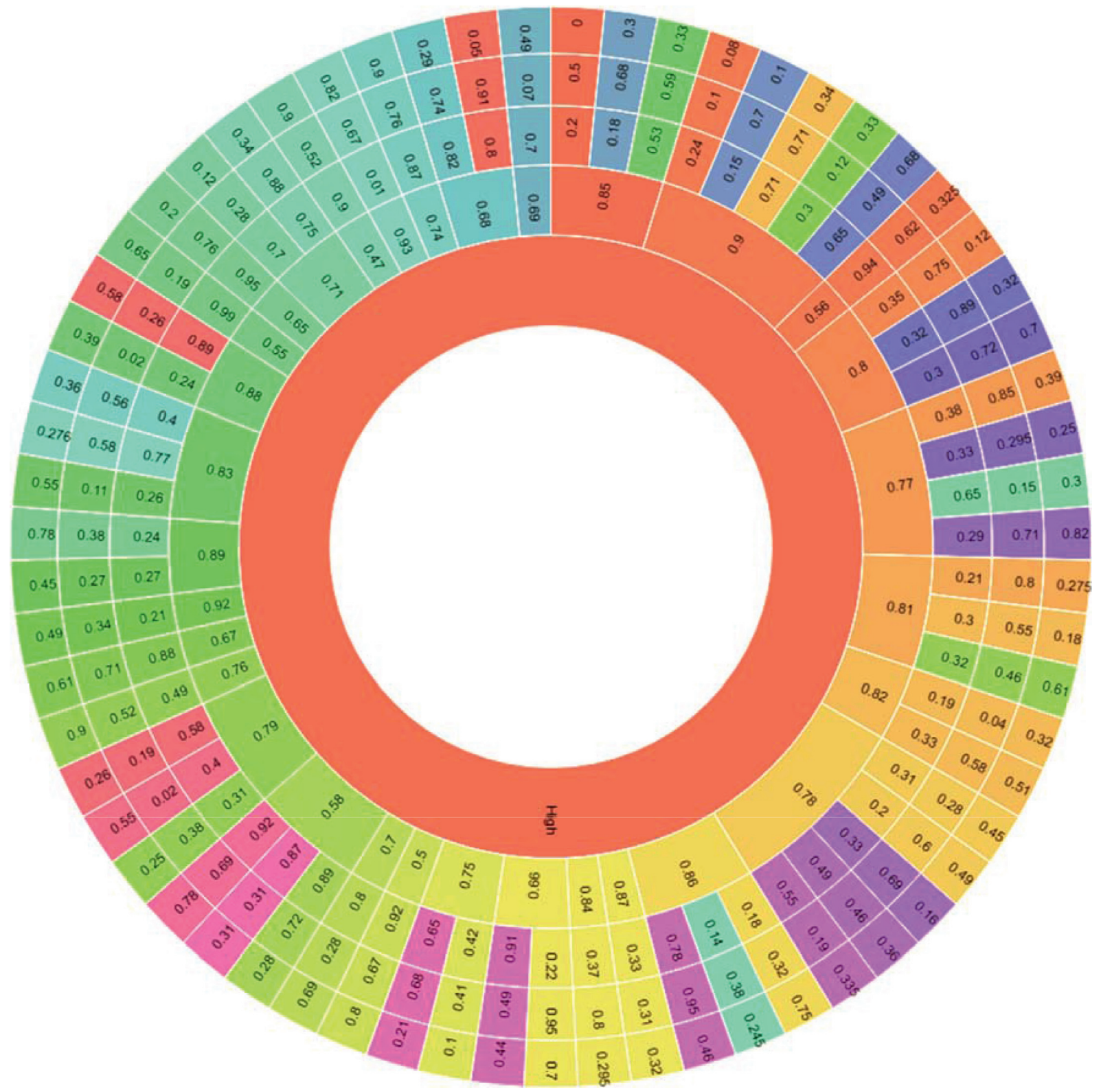

FIgURE 14: Visualization of the class distribution "high."

defuzzMethod: "centroid"

impMethod: "min”

aggMethod: "max"

input: $[1 \times 5$ struct $]$

output: $[1 \times 1$ struct]

After that, the input values of each attribute were passed in order to get the decision. The following is an example of the input format:

$>>$ out $=$ evalfis $\left(\left[\begin{array}{lllll}0.08 & 0.08 & 0.1 & 0.1 & 0.1\end{array}\right]\right.$, fismat $)$

out $=0.5000$
$>>$ out $=$ evalfis $\left(\left[\begin{array}{lllll}0.2 & 0.01 & 0.2 & 0.1 & 0.2\end{array}\right]\right.$, fismat $)$

out $=0.5000$

$>>$ out $=$ evalfis $\left(\left[\begin{array}{lllll}0.7 & 0.8 & 0.7 & 0.9 & 0.6\end{array}\right]\right.$, fismat $)$

out $=0.9044$

$>>$ out $=$ evalfis $\left(\left[\begin{array}{lllll}0.5 & 0.8 & 0.5 & 0.9 & 0.6\end{array}\right]\right.$, fismat $)$

out $=0.5047$

> > out $=$ evalfis $\left(\left[\begin{array}{lllll}0.5 & 0.8 & 0.5 & 0.6 & 0.6\end{array}\right]\right.$, fismat $)$

out $=0.5000$

$>>$ out $=$ evalfis $\left(\left[\begin{array}{lllll}0.9 & 0.8 & 0.5 & 0.6 & 0.9\end{array}\right]\right.$, fismat $)$

out $=0.9044$ 


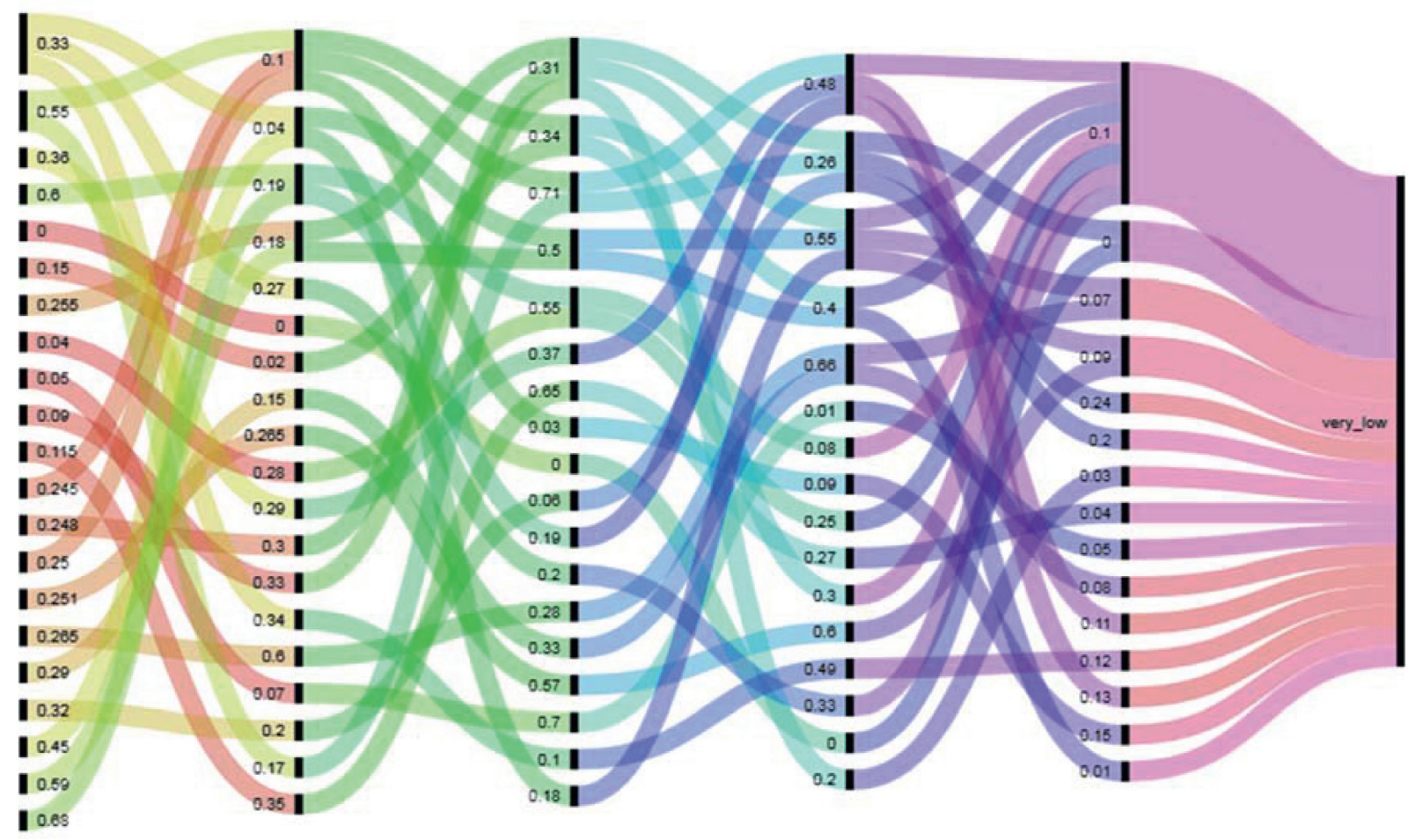

FIgURE 15: Visualization of class distribution "very_low."

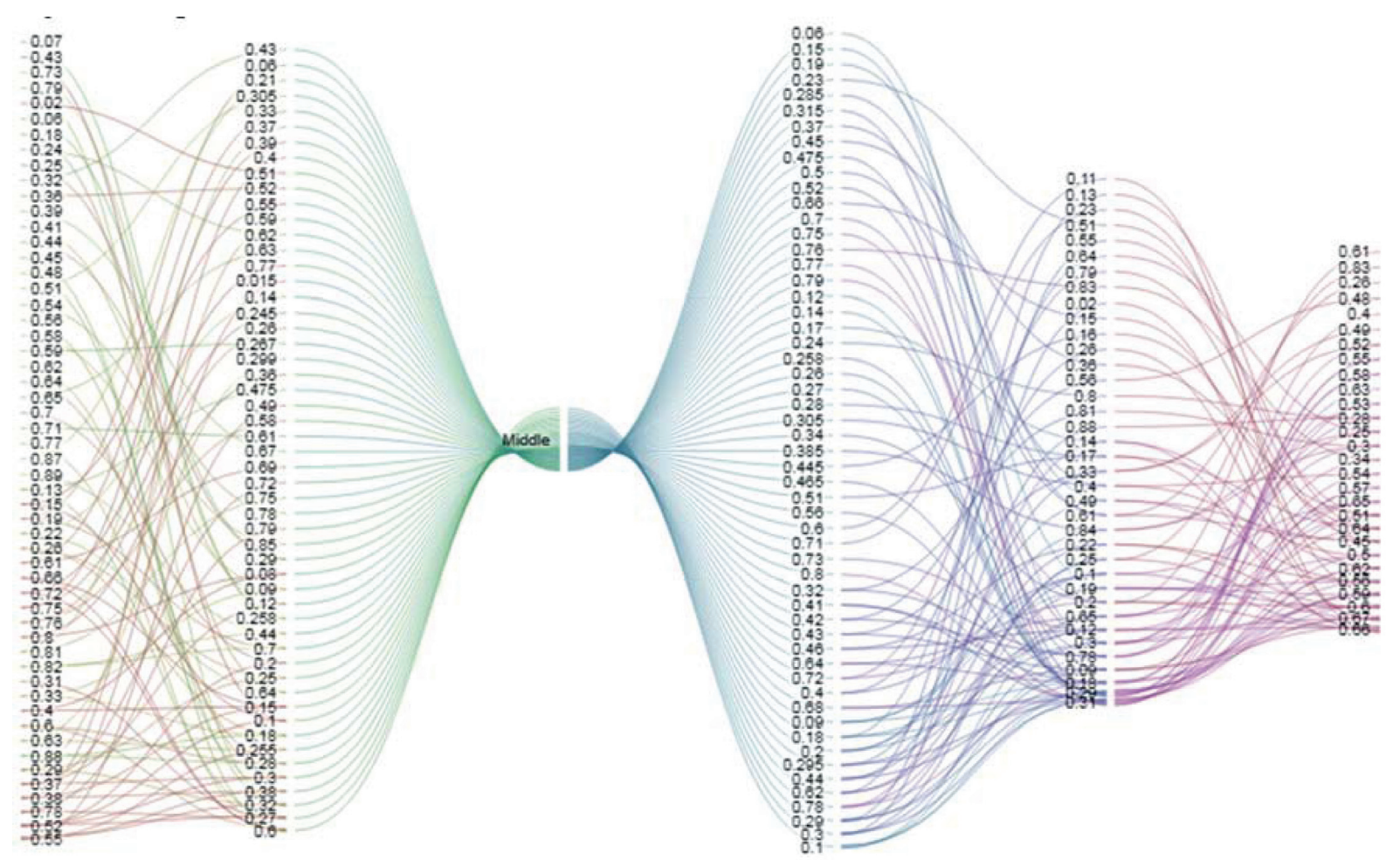

FIGURE 16: Visualization of class distribution "middle." 


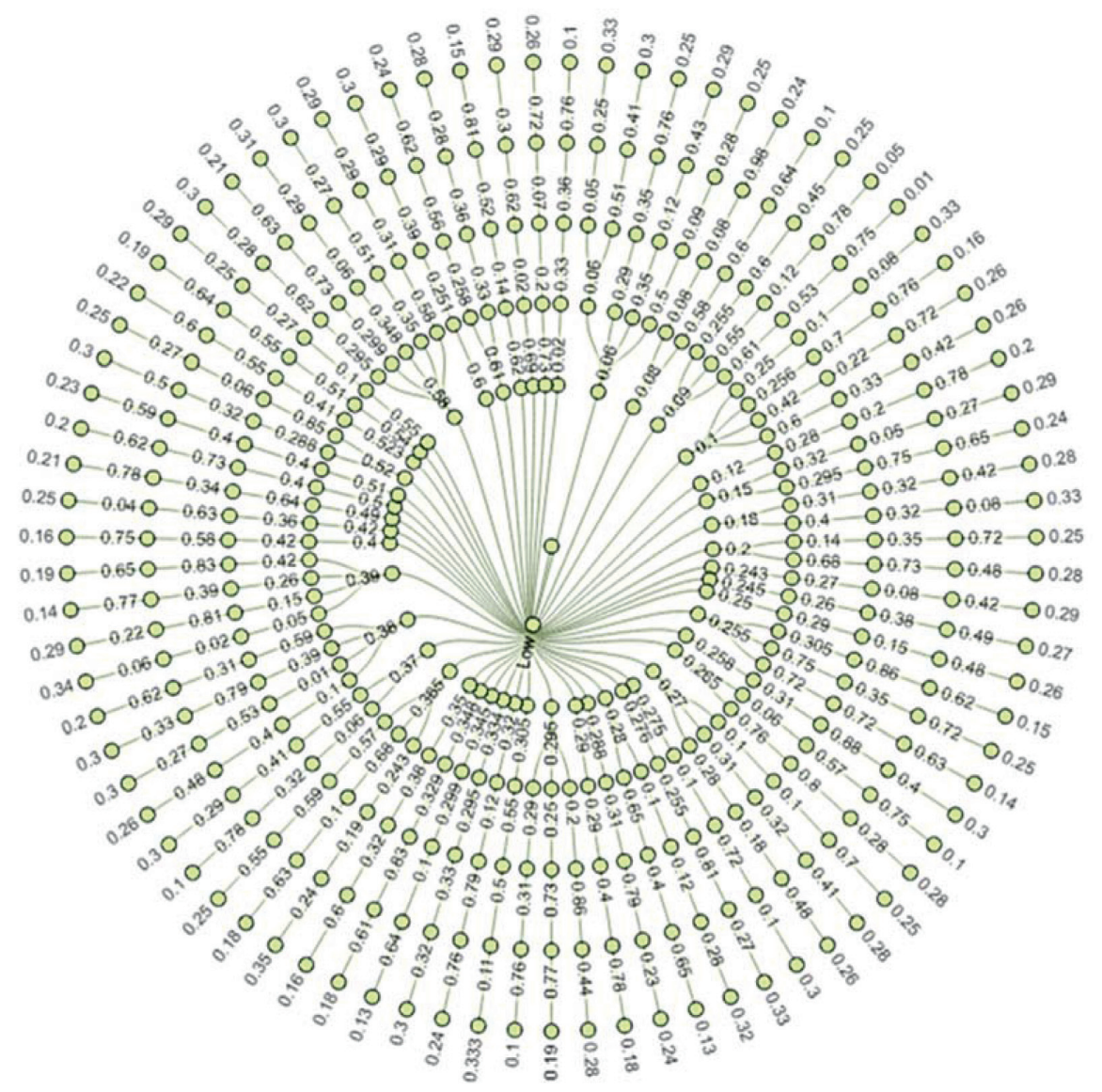

FIgURE 17: Visualization of class distribution "low."

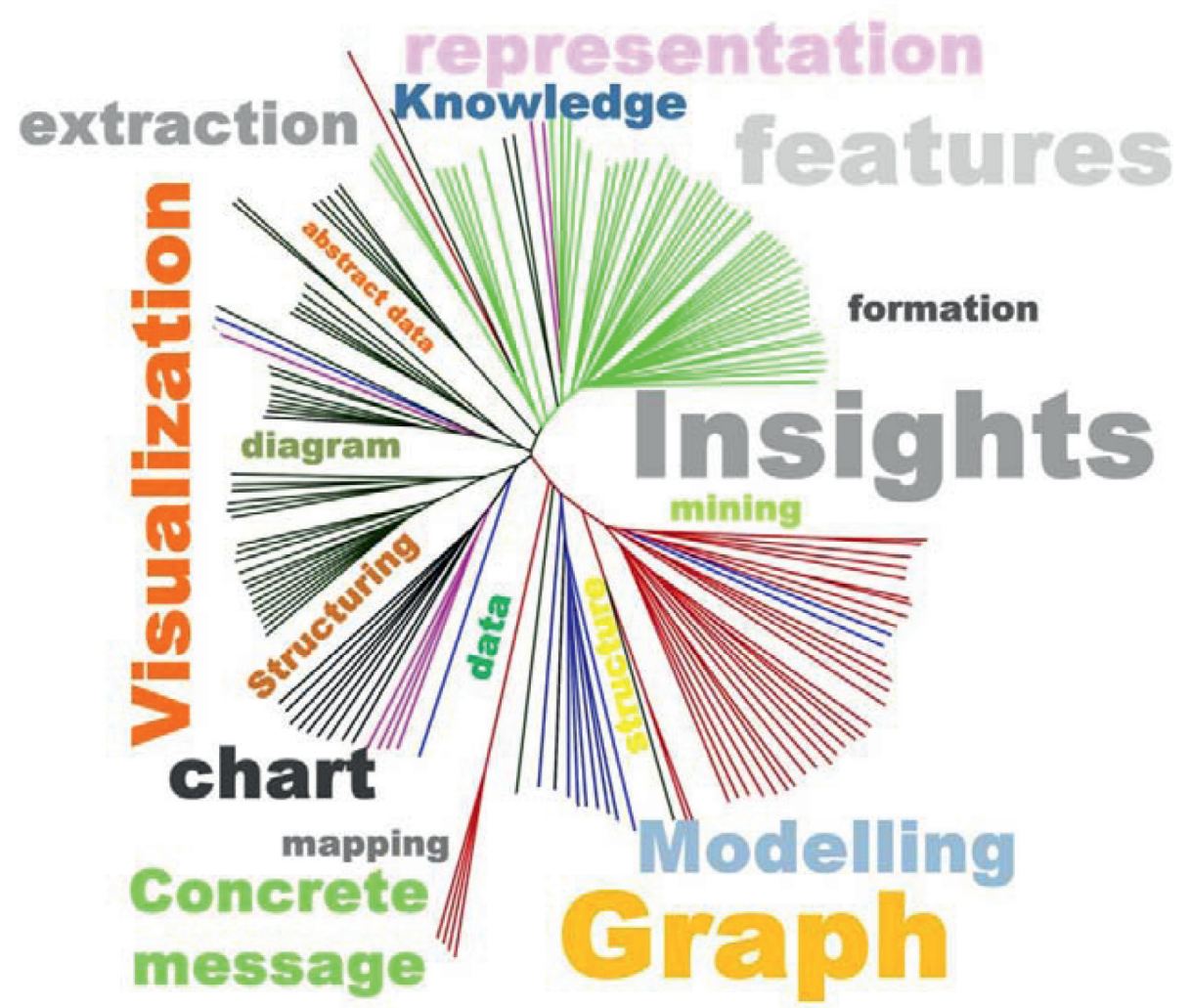

Figure 18: Common terminologies used for visualization. 


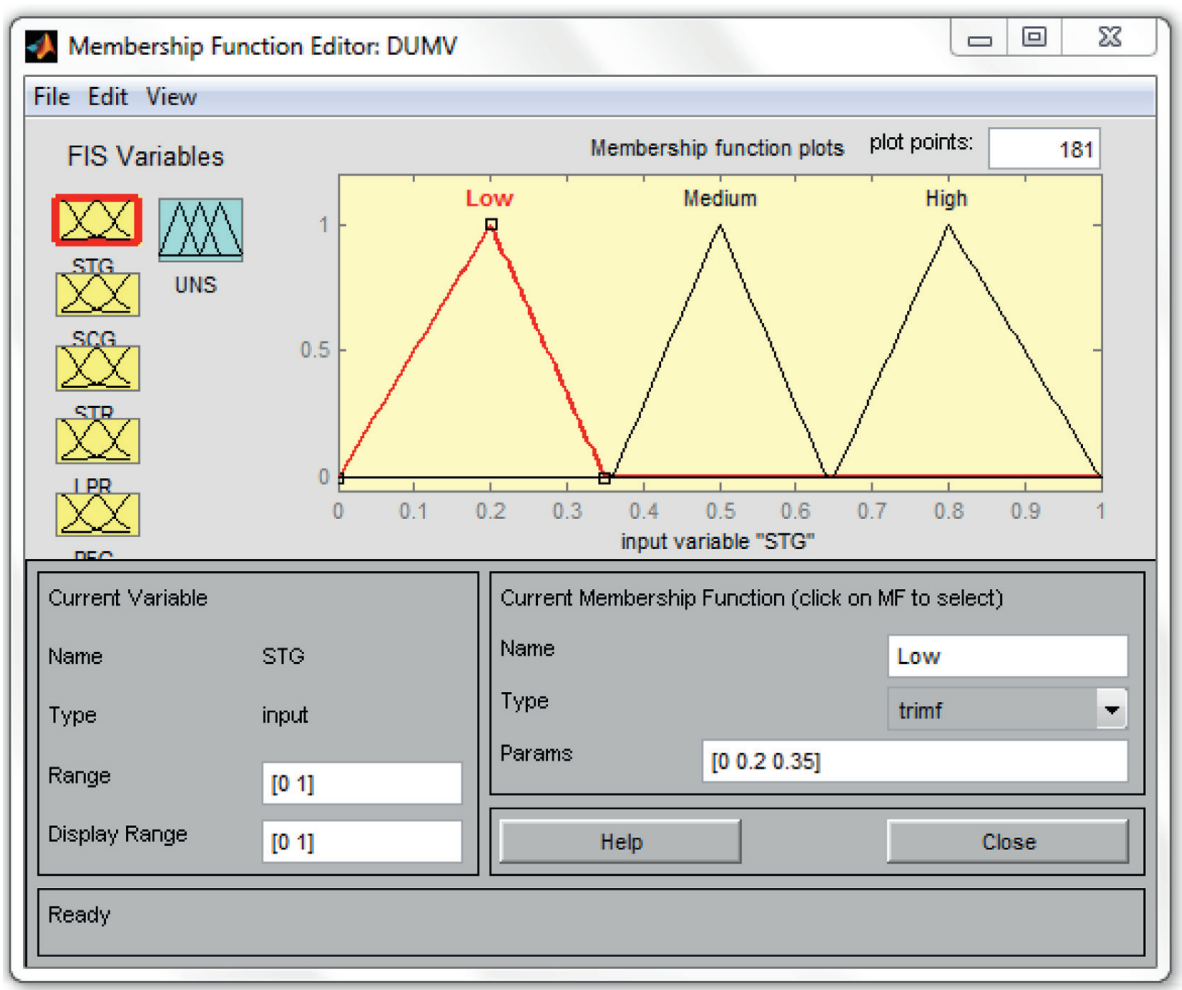

FIGURE 19: Input and output plotting process of the proposed model along with the mf.

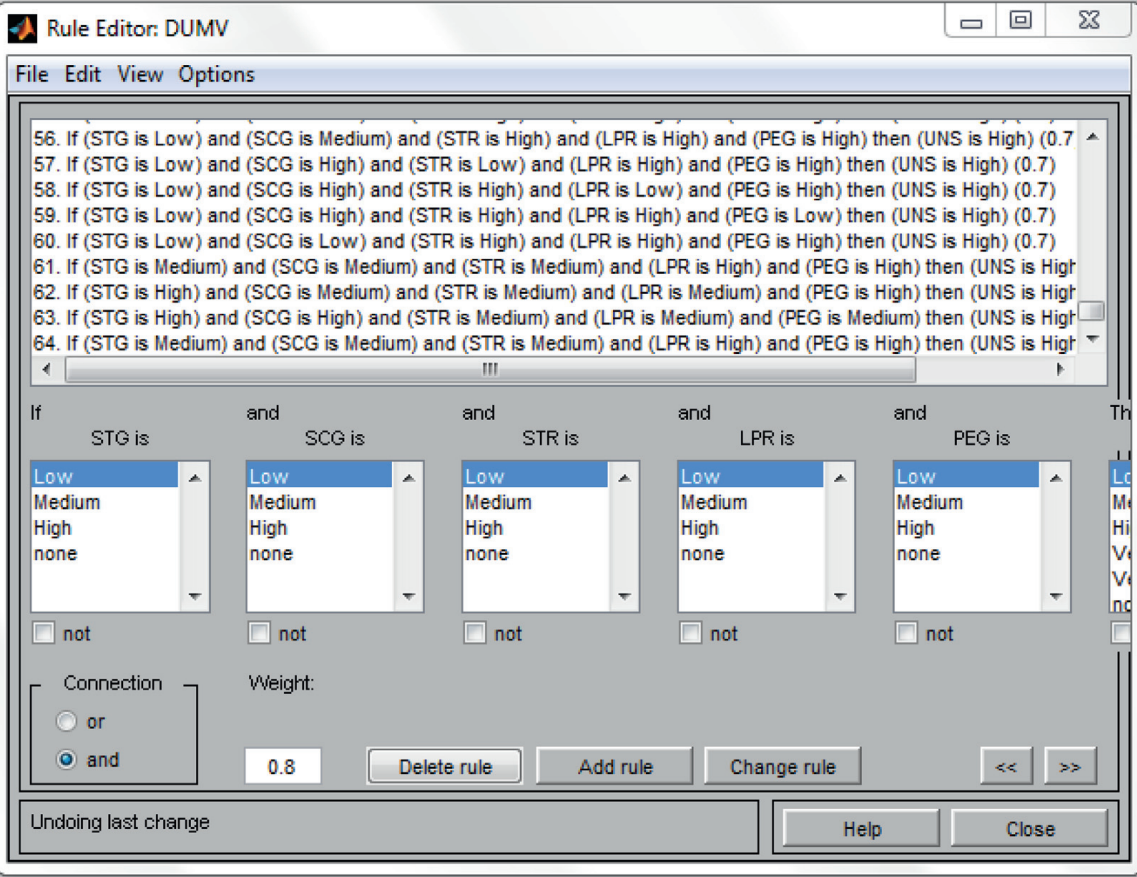

Figure 20: Rule editor of the proposed system. 


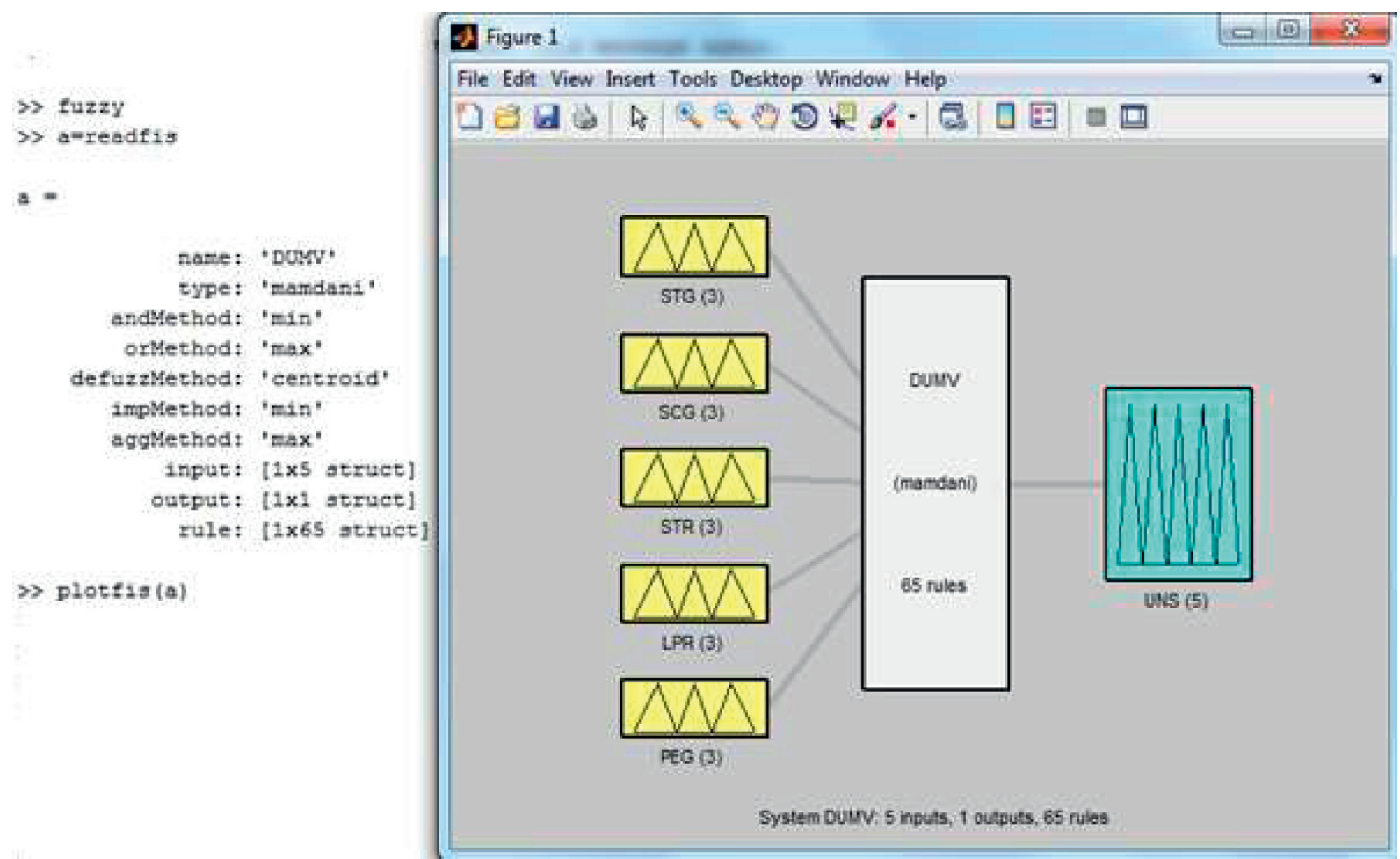

FIgURE 21: Proposed model obtained from the plotting of the $\mathrm{mf}$ and designing of rules.

TABle 1: Proposed model description.

showfis(a)

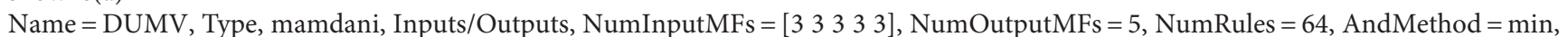
OrMethod $=\max$, ImpMethod $=\min$, AggMethod $=\max$, DefuzzMethod $=$ centroid, InLabels $=$ STG, SCG, STR, LPR, PEG,

OutLabels $=$ UNS, InRange $=\left[\begin{array}{l}0 \\ 1\end{array}\right]$, InMFLabels $=$ Low, Medium, High, OutMFLabel Low, Medium, High, Very_low, Very_high, InMFTypes $=$ trimf

\section{Conclusion}

The data and information are the dire needs of modern day technology and real life. Both data and information are rapidly increasing with the passage of time. It becomes very difficult to understand all the things at the same time. The user needs meaningful extraction from the knowledge of data and information. Visualization plays an important role to extract meaningful information and insights from it. The proposed study is an endeavour toward the user knowledge, data modelling, and visualization by handling through the fuzzy logic-based approach. Fuzzy logic deals with uncertainty and vagueness when they arise in the data. Experimental setup of the proposed research is validated through the data user modelling dataset available in the UCI web repository. Model of the fuzzy inference system was designed based on the inputs, mf, output, and fuzzy rules. The results show that the model is effective and efficient in situations where uncertainty and complexity arise.

\section{Data Availability}

No data were used to support this study.

\section{Conflicts of Interest}

The authors declare that there are no conflicts of interest regarding the publication of this paper.

\section{Acknowledgments}

This research was supported by Science and Technology Project of State Grid Xizang Electric Power Co., Ltd (SGXZJY00JHJS2000007) (Influence of Energy Storage Technology Application on Power Grid) and Science and Technology Project of State Grid Zizang Electric Power Co., Ltd (SGXZJY00JHJS2000008) (Research Technology Service of Multi-Energy Complementary Demonstration Application).

\section{References}

[1] R. Bellazzi, "Big data and biomedical informatics: a challenging opportunity," IMIA Yearbook of Medical Informatics, vol. 22, no. 9, pp. 8-13, 2014.

[2] IDC, "Analyze the future," 2014, http://www.idc.com/.

[3] M. Hilbert and P. López, "The world's technological capacity to store, communicate, and compute information," Science, vol. 332, no. 6025, pp. 60-65, 2011. 
[4] S. D. Sagiroglu, "Big data: a review," in Proceedings of the International Conference on Collaboration Technologies and Systems (CTS '13), pp. 42-47, San Diego, CA, USA, May 2013.

[5] M. Chen, D. Ebert, H. Hagen et al., "Data, information, and knowledge in visualization," IEEE Computer Graphics and Applications, vol. 29, no. 1, pp. 12-19, 2009.

[6] K. Pantazos and K. Pantazos, Custom visualization without real programming, $\mathrm{PhD}$ Thesis, University of Copenhagen, IT University Of Copenhagen, Copenhagen, Denmark, 2012.

[7] H. T. Kahraman, I. Colak, and S. Sagiroglu, "Developing intuitive knowledge classifier and modeling of users' domain dependent data in web, knowledge based systems," 2013, https://archive.ics. uci.edu/ml/datasets/User+Knowledge+Modeling.

[8] A. K. Sahu and P. Dwivedi, "Knowledge transfer by domainindependent user latent factor for cross-domain recommender systems," Future Generation Computer Systems, vol. 108, pp. 320-333, 2020.

[9] J. Chang and J. Hwang, "The role of media in user participation: focusing on the knowledge activity in online space," Telematics and Informatics, vol. 51, 2020.

[10] S. Flowers and M. Meyer, "How can entrepreneurs benefit from user knowledge to create innovation in the digital services," Journal of Business Research, 2020, In press.

[11] J. Constant, "Knowledge visualization and nano-crystal modeling geometry," Applied Surface Science, vol. 473, pp. 668-672, 2019.

[12] F. Desimoni and L. Po, "Empirical evaluation of Linked Data visualization tools," Future Generation Computer Systems, vol. 112, pp. 258-282, 2020.

[13] W. Huang, J. Luo, T. Bednarz, and H. Duh, "Making graph visualization a user-centered process," Journal of Visual Languages \& Computing, vol. 48, pp. 1-8, 2018.

[14] I. C. S. Silva, G. Santucci, and C. M. D. S. Freitas, "Visualization and analysis of schema and instances of ontologies for improving user tasks and knowledge discovery," Journal of Computer Languages, vol. 51, pp. 28-47, 2019.

[15] R. Yu and L. Shi, "A user-based taxonomy for deep learning visualization," Visual Informatics, vol. 2, no. 3, pp. 147-154, 2018.

[16] W. Luo, "User choice of interactive data visualization format: the effects of cognitive style and spatial ability," Decision Support Systems, vol. 122, Article ID 113061, 2019.

[17] G. B. Gebremeskel and B. H. B. Biazen, "Architecture and optimization of data mining modeling for visualization of knowledge extraction: patient safety care," Journal of King Saud University-Computer and Information Sciences, 2019, In press.

[18] S. Al-Dohuki, F. Kamw, Y. Zhao, X. Ye, J. Yang, and S. Jamonnak, "An open source trajanalytics software for modeling, transformation and visualization of urban trajectory data," in Proceedings of the 2019 IEEE Intelligent Transportation Systems Conference (ITSC), pp. 150-155, Auckland, New Zealand, October 2019.

[19] W. A. C. Rojas and C. M. Villegas, "Graphical representation and exploratory visualization for decision trees in the KDD process," in Proceedings of the 2012 XXXVIII Conferencia Latinoamericana En Informatica (CLEI), pp. 1-10, Medellin, Colombia, October 2012.

[20] B. Macek and M. Atzmueller, "Visualizing the impact of time series data for predicting user interactions," in Proceedings of the 2013 IEEE/ACM International Conference on Advances in Social Networks Analysis and Mining (ASONAM 2013), pp. 1477-1478, Niagara Falls, ON, Canada, August 2013.
[21] F. Giunchiglia, S. R. Ojha, and S. Das, "SemUI: a knowledge driven visualization of diversified data," in Proceedings of the 2017 IEEE 11th International Conference on Semantic Computing (ICSC), pp. 234-241, San Diego, CA, USA, January 2017.

[22] S. F. H. Li, S. Mukhopadhyay, A. J. Saykin, and L. Shen, "Interactive machine learning by visualization: a small data solution," in Proceedings of the 2018 IEEE International Conference on Big Data (Big Data), pp. 3513-3521, Seattle, WA, USA, December 2018.

[23] M. A. Rafi, "Visualization of multi-dimensional resource space," in Proceedings of the 2017 13th International Conference on Semantics, Knowledge and Grids (SKG), pp. 182187, Beijing, China, August 2017.

[24] M. Risi, M. I. Sessa, M. Tucci, and G. Tortora, "CoDe modeling of graph composition for data warehouse report visualization," IEEE Transactions on Knowledge and Data Engineering, vol. 26, no. 3, pp. 563-576, 2014.

[25] D. Sacha, A. Stoffel, F. Stoffel, B. C. Kwon, G. Ellis, and D. A. Keim, "Knowledge generation model for visual analytics," IEEE Transactions on Visualization and Computer Graphics, vol. 20, no. 12, pp. 1604-1613, 2014.

[26] L. A. Zadeh, "Fuzzy logic," Computer, vol. 21, no. 4, pp. 83-93, 1988.

[27] Fuzzy Logic Tool box ${ }^{\mathrm{TM}}, 2$ User's Guide, The MathWorks, Inc, Apple Hill Drive Natick, MA, USA, 2010.

[28] J. Yen and R. Langari, Fuzzy Logic: Intelligence, Control and Information, Prentice-Hall, Upper Saddle River, NJ, 1st edition, 1999.

[29] S. Nazir, S. Anwar, M. A. Khan, H. Khan, and M. Nazir, “A novel fuzzy logic based software component selection modeling," in Proceedings of the International Conference on Information Science and Application (ICISA), pp. 1-6, IEEE, Suwon, Korea, May 2012.

[30] S. Nazir and M. Nazir, "Comparisons of membership functions for fuzzy rules," VAWKUM Transactions on Computer Sciences, vol. 3, pp. 10-14, 2014.

[31] S. Nazir, S. Shahzad, S. Mahfooz, and M. N. Jan, "Fuzzy logic based decision support system for component security evaluation," International Arab Journal of Information and Technology, vol. 15, pp. 1-9, 2015.

[32] S. Nazir, S. Shahzad, I. Zada, and H. Khan, "Evaluation of software birthmarks using fuzzy analytic hierarchy process," in Proceedings of the Fourth International Multi-topic Conference, pp. 171-175, Jamshoro, Pakistan, October 2015. 